Protocol design and ideas research exchange

\title{
Influence of the hydration on autonomic modulation and cardiorespiratory parameters of coronary heart disease patients submitted to a cardiovascular rehabilitation session: crossover clinical trial protocol
}

\author{
Anne Kastelianne França da Silva ${ }^{1}$ (D), Maria Júlia Lopez Laurino ${ }^{1}$ (D), Laís Manata Vanzella ${ }^{1}$ (D), \\ Lorena Altafin Santos $^{1}$ (D), Felipe Ribeiro ${ }^{1}$ (D), Dayane Andrade Genoni Corazza ${ }^{1}$ (D), \\ Luiz Carlos Marques Vanderlei ${ }^{1}$ \\ ${ }^{I}$ Universidade Estadual Paulista, Faculdade de Ciências e Tecnologias, Programa de Pós- \\ Graduação em Fisioterapia, Presidente Prudente, SP, Brasil.
}

\begin{abstract}
Aims: The influence of fluid replacement, realized during and after the exercise on individuals with coronary artery disease (CAD) remains poorly understood. To investigate the influence of hydration on cardiac autonomic modulation, cardiorespiratory parameters and perceived exertion and discommodity, of coronary heart patients submitted to cardiac rehabilitation (CR) session. Methods: This cross-over clinical trial, will recruit 31 adults with more than 45 years old, participants of a cardiovascular rehabilitation program, with CAD diagnosis. The participants will be submitted to an experimental protocol composed of three phases: I) Maximal stress test; II) Control protocol (CP); and III) Hydration protocol (HP). The CP and HP will consist of 10 min of rest in a supine position, 15 min of warming, $40 \mathrm{~min}$ of treadmill exercise, $5 \mathrm{~min}$ of cooling down and $60 \mathrm{~min}$ of rest in a supine position. In the HP, the participants will be hydrated with mineral water, based on the bodyweight reduction of the CP. The water intake will be divided into eight equal portions, offered during the treadmill exercise and recovery period. On CP and HP will be evaluated linear and nonlinear indices of heart rate variability, the heart rate, systolic blood pressure, diastolic blood pressure, respiratory rate, oxygen partial saturation, perceived exertion and discommodity on specifics moments. Conclusion: The results of this study will allow us to identify if the proposed protocol will be able to positively influence the outcomes and, consequently, if could be implement in the clinical practice.
\end{abstract}

Keywords: autonomic nervous system, aerobic exercise, recovery, hydration.

\section{Introduction}

The exercise practice is associate to innumerous physical and mental benefits ${ }^{1}$ in many populations ${ }^{2-5}$ that include individuals with cardiovascular disease (CVD) ${ }^{6}, 7$, and the cardiovascular rehabilitation programs specifically, can also promote many benefits for health, like positive changes in the cardiorespiratory and autonomic system ${ }^{8-11}$. However, during the physical exercise realization, the body temperature is high and, consequently, an increase in the transpiration rate occurs, which can promote liquid loss and if the answer is not correctly can promote a dehydrate state ${ }^{12}$. This liquid loss induced for exercise, causes injuries on cardiovascular and autonomic systems ${ }^{13-15}$ which can compromise the recovery capacity and cardiovascular health ${ }^{16,17}$.

In this sense, different strategies to accelerate the autonomic and cardiovascular system recovery after physical exercise practice have been studied on literature ${ }^{15-20}$, and water intake is one of than ${ }^{21-23}$. Studies that investi- gated the effects of water intake associated to physical exercise, pointed that this strategy was able to attenuate cardiovascular $^{18,24}$ and autonomic ${ }^{20-23}$ alterations promoted by the thermic stress as a result of physical exercise and to promote a faster recovery of the organism ${ }^{18,20-23}$.

However, studies that approach this thematic, used different strategies, as the type of the drink (water ${ }^{21-23}$ and/or drinks with electrolytes or carbohydrates ${ }^{14-18,20}$ ), the moment (during ${ }^{17}$ and/or after the exercise ${ }^{14-16,18,20-}$ ${ }^{24}$ ), the quantity of the hydric ingestion (single dose $\mathrm{e}^{16,22,23}$ or fractional dose $\mathrm{e}^{14,15,17,18,20,21}$ ), the exercise intensity (maximum $^{22}$ and/or submaximum ${ }^{14,15,17,18,20,21,23,24}$ ), exercise duration $\left(30^{23}, 45^{16}\right.$ to $90^{14,15,17,18,20,21}$ minutes), the type of the proposed exercise (resistance ${ }^{16,22}$ or aerobic $\left.^{14,15,17,18,20,21,23,24}\right)$ and recovery time $\left(30^{22,23}, 45^{17}\right.$, $60^{14-16,18,20,21}$ or 4 hours $^{24}$ ). Furthermore, this thematic was investigated on health young and/or athletes with a sample that varied between 5 and 31 individuals ${ }^{14-18,20-24}$. However, the absence of studies that investigate these aspects on individuals with CVD, known for presenting 
autonomic and cardiovascular conditions at rest different of healthy individuals ${ }^{25,26}$, needs to be highlighted.

In this sense, we understand how much relevant is to submit individuals with coronary artery disease to a hydration protocol associate to a conventional model of rehabilitation session, to determine if the model of the proposed exercise can promote liquid loss, if this loss can influence the cardiovascular and autonomic parameters of individuals with coronary artery disease, and if the hydration is also a protector factor of the cardiovascular and autonomic system, how was observed in healthy individuals ${ }^{13,21,27}$. Furthermore, we could evaluate if the hydration influences cardiorespiratory parameters and the subjective perceived exertion/pain as well as if we could use these findings as predictor factors during the clinical practice.

Therefore, the objective of this study aimed to investigate the influence of the hydration on cardiac autonomic modulation, through heart rate variability (linear and nonlinear indices), cardiorespiratory parameters (heart rate, respiratory rate, oxygen partial saturation, systolic blood pressure, diastolic blood pressure), subjective perceived exertion and subjective perceived pain, of coronary artery disease patients submitted to a moderate-intensity aerobic exercise. The hypothesis is that these individuals will be beneficiated by restoring the liquid, improving the autonomic answer, cardiorespiratory parameters, and subjective perceived exertion and pain during the exercise and recovery time.

\section{Material and Methods}

\section{Study design and participants}

The present protocol is a crossover clinical trial, registered prospectively on ClinicalTrials.gov (NCT 03198806) and follows the SPIRIT $2013^{28}$ and TIDieR ${ }^{29}$ recommendations for clinical trials.

The participants of this study will be recruited in two sectors of cardiovascular rehabilitation, in the city of Presidente Prudente, SP, Brazil. The inclusion criteria will be:

- Male participants with more than 45 years old

- A medical diagnosis of ischemic coronary artery disease, with left ventricular function preserved, that corresponds to an ejection fraction of left ventricle higher than $50 \%$, confirmed by echocardiogram indexed previously in their medical record

- Do not have a recent history of smoking and/or alcoholism

- To be performing cardiovascular rehabilitation program for at minimum 3 months previously the data collection

- Do not have historic of infections, unstable angina, uncontrolled hypertension, significates valvar disease and/or uncontrolled metabolic disease (for example, uncontrolled diabetes and thyroid disease) during the recruitment period.

Exclusion criteria will be when at least one of the following characteristics after the beginning of the protocol:

- Abnormal hemodynamic answers during the exercise, like heart rate and/or blood pressure increase/fall disproportionate with lower/higher load levels.

- Presence of myocardial ischemia and/or several ventricular arrhythmias during the exercise test.

- Temporal series of RR intervals with less than $95 \%$ of sinus beat ${ }^{30}$

- Nonattendance at least one of the protocol phases

Sample size

The sample size was defined by the online software contained on the website http://www.lee.dante.br/, based on RMSSD index. The magnitude of the significant difference assumed was $12 \mathrm{~ms}$, considering a standard deviation of $16 \mathrm{~ms}^{15}$, with an alpha risk of $5 \%$ and a beta of $80 \%$, the sample size resulted in 28 participants. Considering possible sample losses will be increased by $10 \%$ of de sample size calculated, totalizing 31 participants.

\section{Ethic aspects}

The participants will be informed about the procedures and the aims of this study and after agreeing to participate, signed a written informed consent form. This study was approved by the Committee for Ethics and Research of the Faculty of Science and Technology FCT/ UNESP (CAAE: 54864716.8.0000.5402) and followed the Helsinki declaration.

\section{Design of the experimental approach}

The participants will be submitted individually to an experimental procedure realized on a treadmill, with a minimum of 48 hours of interval, to permit their recovery. The experimental procedure will be divided into three following phases, with a duration of one day each (Figure 1):

I. Maximum stress test: will be performed to determine the peak oxygen consumption $\left(\mathrm{VO}_{2}\right.$ peak), to define the used load in the following phases:

II. Control protocol (CP): the participants will remain 10 minutes at rest position followed by 15 minutes of warming, with activities involving stretching and global exercises, 40 minutes of physical exercise in a treadmill, 5 minutes of cooling down and 60 minutes of recovery. In this phase, the participants will not be hydrated.

III. Hydration protocol (HP): the participants will perform the equal $\mathrm{CP}$ activities, but with the administration of mineral water (Bonafont, Brazil) that will be divided into 8 portions uniformly, administered for 10 minutes in regular intervals from the $10^{\text {th }}$ minute of the exercise until the lasts 25 minutes of the recovery period. 


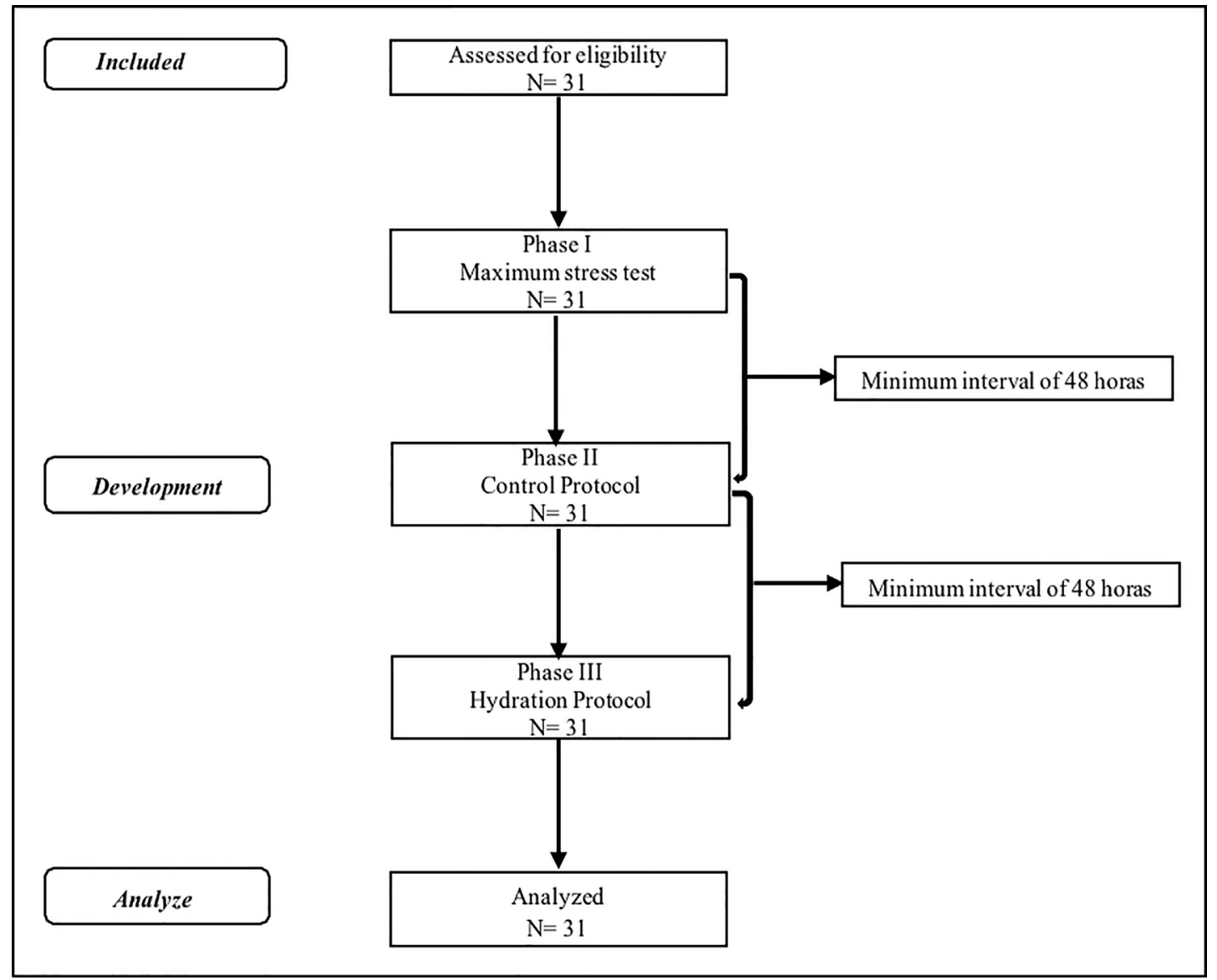

Figure 1 - Protocol design.

\section{Previous orientation to the experimental procedure}

Aiming for the reliability of the results, the participants will receive orientations to comply with the experimental procedure phase, as state below:

- Not to ingest caffeine-based drinks (coffee, chocolate, soda and tea) for 24 hours prior to all study phases;

- Consume light meal for two hours prior all the study protocol;

- Wear comfortable clothes appropriate for exertion, such as shorts, shirt, shoes, and socks;

- To maintain their habitual physical activities during the protocol development, but to avoid vigorous physical exertion the day before;

- To intake $500 \mathrm{~mL}$ of water two hours before the beginning of the phases II (CP) and III (HP) ${ }^{30}$, to guarantee the basal hydration condition of all participants.

\section{Details of the experimental procedure}

The details of the experimental procedure are described below and the Figure 2 illustrates the protocols activities.

\section{Phase I: Maximum stress test}

For definition of the exercise load used in the protocols, the participants will perform in a treadmill (Super $A T L$, Inbrasport, Brazil) a maximum stress test, in accordance with the maximal Bruce protocol ${ }^{31}$ on Laboratório de Fisiologia Celular do Exercício (LaFICE) at Faculdade de Ciências e Tecnologia - UNESP/PP.

The participants will remain at rest on the treadmill in orthostatic position to stabilization of the initial values and after that, will begin the maximum stress test, with 5 minutes of warming and $2.5 \mathrm{~km} / \mathrm{h}$ of speed, followed by the maximal Bruce protocol, that consist in progressive incremental of load through the tilt and speed on every three minutes. Verbal encouragement will be used to achieve the maximum physical exertion, with the test being interrupted by the participant exhaustion or when the maximum stress test was achieved ${ }^{31}$. All participants will be continuously monitored during the rest and the exercise until exhaustion, and for $5 \mathrm{~min}$ of recovery through an electrocardiogram. Aiming the safety of the participants, all the procedures related to the incremental test will be supervised by a Cardiologist with expertise in maximum stress test. 


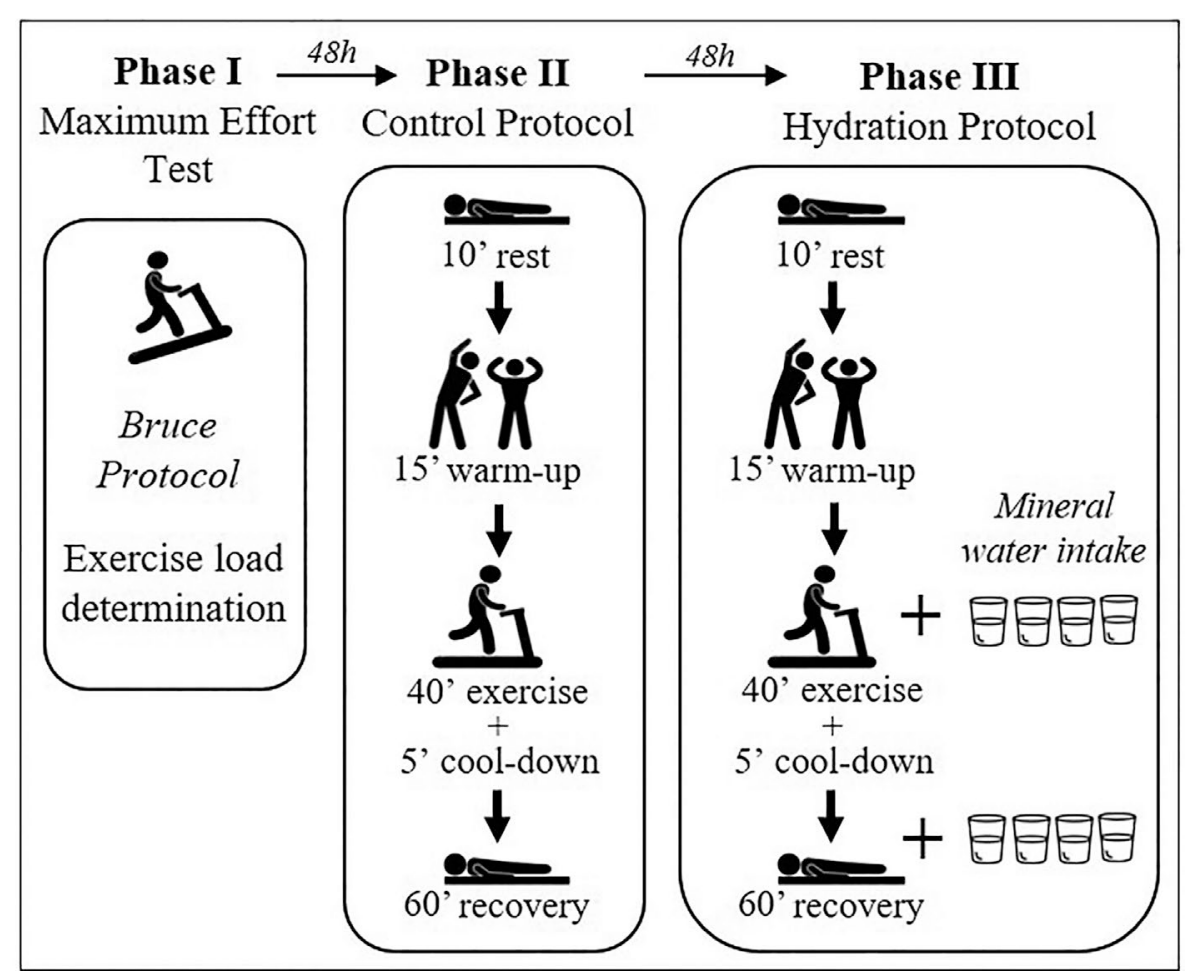

Figure 2 - Graph abstract of the experimental procedure.

To determine the oxygen consumption $\left(\mathrm{VO}_{2 \text { peak }}\right)$, exhaled gases will be analyzed using a commercial system Quark PFT, (Cosmed, Rome, Italy), calibrated with volumes and gases of known concentration. The $\mathrm{VO}_{2 \text { peak }}$ will be considered the highest $\mathrm{VO}_{2}$ reached in the test. The used load in the CP and HP will be $60 \%$ to $80 \%$ of the maximum heart rate (HR) reached at the anaerobic threshold (AT) of the test. For individuals that do not reach the AT during the test, the used load will be $60 \%$ to $80 \%$ of the $\mathrm{HR}$ value reached in $\mathrm{VO}_{2 \text { peak. }}$. For the determination of HR, the participant will wear a HR monitor (RS800CX, Polar Electro Oy, Finland) and the beat-to-beat will be recorded $^{32}$ and subsequently, the values obtained will be extracted.

\section{Phases II and III: Control and hydration protocol}

The protocols will be realized on CEAFiR of Faculdade de Ciências e Tecnologia - UNESP, between 01h30 a. $\mathrm{m}$ to $6 \mathrm{~h} 00 \mathrm{p}$.m. to avoid circadian variations. Temperature (temperature between $22{ }^{\circ} \mathrm{C}$ to $25^{\circ} \mathrm{C}$ ) and the humidity (humidity between 40 to $60 \%$ ) will be controlled. To reduce the anxiety of the participants during the tests, a small number of people will stay on the data collection locally. During this phase, researchers with expertise in the ambulatory phase of cardiovascular physical therapy will supervise all the procedures of the study.

Initially, in both protocols, the participants will be oriented to collect a urine sample despising the first jet to analyze the degree of hydration of the participants. In the hydration condition, it will be verified: bodyweight, though a digital scale (Plenna, TIN 00139 MAXIMA, Brazil) and height, though a stenographer (ES 2020 Sanny, Brazil).

Before the beginning of the protocols (CP and HP) will be placed on the participants a HR monitor (RS800CX, Polar Electro Oy, Finland) to record the heart rate beat-to-beat during the protocols and the participants will remain lying comfortably for 10 minutes. At the final of 10 minutes of rest, the axillar temperature will be measured through a Thermometer (BD Thermofácil, China). The cardiorespiratory parameters Heart Rate (HR), Systolic Blood Pressure (SBP), Diastolic Blood Pressure (DBP), respiratory rate (f), Oxygen partial saturation (SpO2), Subjective perceived exertion (SPE) and subjective perceived pain (SPP) will be also measured at this moment.

After these measures, the participants will realize 15 minutes of warming, with activities involving stretching and global exercise, 40 minutes of treadmill (intensity of $60 \%$ to $80 \%$ to $\mathrm{VO}_{2 \text { peak }}$ or AT) and 5 minutes of cooling down with a half of load used on the previous exercise. During the exercise period on a treadmill, new measurements of the parameters: HR, SBP, DBP, SpO2, SPE, SPP in minutes 15 and 35 will be realized.

At the end of the activity (rest + warming + treadmill exercise + cooling down), on the $70^{\text {th }}$ minute, the axillar temperature of the individuals will be measured again with than at rest, lied in a calm environment and 
monitored for further 60 minutes. During this period, it will be analyzed the HR, SBP, DBP, SpO2, SPE, SPP parameters on $1^{\text {st }}, 3^{\text {rd }}, 5^{\text {th }}, 7^{\text {th }}, 10^{\text {th }}$ and every 10 minutes from this, until the end of the recovery.

The RR intervals will be analyzed on the following moments: M1 (lasts 5 minutes of the rest), M2 $\left(15^{\text {th }}\right.$ to $20^{\text {th }}$ minutes of the exercise), M3 ( $35^{\text {th }}$ to $40^{\text {th }}$ minutes of the exercise), M4 ( 0 to $5^{\text {th }}$ minutes of the recovery), M5 ( $5^{\text {th }}$ to $10^{\text {th }}$ minutes of the recovery), M6 ( $15^{\text {th }}$ to $20^{\text {th }}$ minutes of the recovery), M7 ( $25^{\text {th }}$ to $30^{\text {th }}$ minutes of the recovery), M8 ( $40^{\text {th }}$ to $45^{\text {th }}$ minutes of the recovery) and M9 $\left(55^{\text {th }}\right.$ to $60^{\text {th }}$ minutes of the recovery). For the evaluation of the autonomic modulation will be obtained in this section at least $256 \mathrm{RR}$ consecutive intervals ${ }^{32}$.

In both protocols, after the recovery period, the participants will be oriented to collect a urine sample again in order to analyze its composition and after this, the final body weight measurement will be performed.

\section{Hydration strategy}

The amount of drink administered during the treadmill physical exercise and HP recovery, will be obtained through the body mass loss, evaluated thought the body mass difference verified before of the $\mathrm{CP}$ begins and after their recovery period. The body mass will be measured thought a digital scale (Plenna -TIN 00139 MAXIMA, Brazil). The use of this technic implies that one gram of body mass loss is equivalent to one milliliter of liquid $\operatorname{loss}^{33,34}$. Thus, the body mass loss of each participant indicates the amount that will be administered during the treadmill physical exercise and the HP recovery period.

For the participants hydration, it will be used mineral water without gas (Bonafont, Brazil). The strategy adopted on this protocol will be realized, based on previous studies of the group ${ }^{20,21}$, which will be done in intervals of 10 minutes from the $10^{\text {th }}$ minute of the treadmill physical exercise until the lasts 25 minutes of the recovery period. Therefore, the total amount of ingested will be divided into 8 equal portions.

Furthermore, the hydration dynamic condition before and after the CP and HP performed will be verified through the urine analysis (Combur $10 \mathrm{M}$ teste, Roche®, Brazil) and the specific density will be used as a marker of the hydration degree ${ }^{33}$. For the performed of this procedure, the participants will be instructed to collect their urine previously to $\mathrm{CP}$ and HP and after the recovery period, despising the first jet. The participants that have a specific density under of 1.020 will be considered hydrated and those with specific density above, dehydrated ${ }^{35}$.

\section{Assessed outcomes}

\section{a. Primary outcome}

- Heart rate variability: The heart rate variability (HRV) analysis will be realized from the recorded data through a HR monitor Polar RS800CX
(Polar Electro, Kempele, Finland) ${ }^{36}$ through linear methods, that will be analyzed on time(SDNN and RMSSD), frequency domain (LF, $\mathrm{HF}$ and LF/ $\mathrm{HF}$ ), geometric indices (SD1, SD2 and SD1/SD2) and nonlinear methods (Detrended Fluctuation Analysis (DFA), recurrence rate (REC), determinism (DET) and approximated entropy $(\mathrm{ApEn})^{32,37-39}$.

The HRV indices will be obtained from the Kubios HRV software -2.0 version $^{40}$, except DFA indices that will be calculated by an available software on PhysioNet (http:// www.physionet.org/), an online forum that collects biomedical and signal records and software to analyze this signals ${ }^{41}$. For this analyze, temporal series of RR intervals will be initially submitted to digital filtering from the Polar Pro Trainer software (5.0 version) complemented by manual, for the elimination of the ectopic, premature and artefacts beats, and just series with more than $95 \%$ of the sinus beat will be included in the study ${ }^{30}$. From the visual analysis of the temporal series will be observed the absence of the artifacts or cardiac arrhythmias that would influence on HRV analyze.

\section{b. Secondary outcomes}

- Blood pressure: The SBP and DBP parameters will be measured, using a stethoscope (Littmann, Saint Paul, USA) and aneroid sphygmomanometer (Welch Allyn - Tycos, New York, USA), according to the VI Brazilian Hypertension Guidelines $^{42}$. The values will be registered in individual file. To avoid errors in blood pressure determination, a trained profissional will measure this parameter during all the experimental.

- Heart rate: The HR will be recorded beat-to-beat through a HR monitor Polar RS800CX (Polar Electro, Kempele, Finland), previously validated equipment to capture this parameter ${ }^{36}$.

- Respiratory rate: The measures will be realized through the respiratory score during one minute, without the volunteer knowledge, to maintain the usual respiration ${ }^{43}$.

- Partial oxygen saturation: The $\mathrm{SpO} 2$ will be verify through a pulse oximeter (Mindray PM-50 Pulse Oximeter, China).

- Subjective perceive exertion: The subjective perceive exertion (SPE or RPE) will be obtained using a Borg scale ${ }^{44}$. The scale will be presented to the participants at a form that they can express the degree of exertion feeling.

- Subjective perceive pain: At the reason of the studied population, will be also evaluated the subjective perceive pain through the Borg CR10 scale $^{44}$, with the objective to evaluate the discommodity degree perceived to the participants during the protocol phases. The Scale comprises 10 points, varying of 0 (absolutely nothing/ with- 
out pain) to 10 (extremely strong/ maximum pain). The participants will be questioned if present some discommodity and in affirmative cases the scale will be presented at a form that they can indicate the score of the discommodity felling.

\section{Blinding, randomization, and allocation}

The draw of the study did not permit the randomization of the groups and consequently the secret allocation since for this execution the participants mandatorily will need to follow the sequence of the study pre-determined (Phase I $\rightarrow$ Phase II $\rightarrow$ Phase III). The first phase is necessary for the definition of the exercise load used in the protocols and only after this phase, the protocol will be applied. The randomization of the other protocols does not allow because the amount of the drink administered during the HP (phase III), that will be obtained through the body mass loss, as described previously.

The blinding (AKFS and LCMV) will be possible to the extraction of the primary outcome data (HRV), HR (secondary outcome) and to statistical analysis, where the researcher will have the assess to the data. To the others secondary variables, it will be not possible the blinding because of the logistic adopted at the study.

\section{Data analyses}

Descriptive statistics will be used to describe the population profile and the results will be presented as mean, median, standard deviation, minimum and maximum, and confidence interval values. The data files will be examined regularly to ensure their integrity and participants will receive an ID to preserve the confidentiality of the data. The normality of the data will be determined by Shapiro-Wilk test. For the variables weight and body temperature, the comparison of the moments of the same protocol will be realized by paired Student's t-test for normal distribution of data or Wilcoxon test for non-normal distribution of data. For the analyze between the protocols unpaired Student's t-test will be used for normal distribution data or Mann-Whitney test for non-normal data. The comparison of the cardiorespiratory parameters, perceived exertion and pain, and HRV indices between the protocols (CP vs HP) and moments (rest vs exercise and rest times vs recovery times) will be made by two-way repeated measures ANOVA. The repeated-measures data will be checked for sphericity violation using Mauchly's test and the Greenhouse-Geisser correction will be conducted when sphericity is violated. For analyzing of the moments (rest $v s$ exercise and rest times vs recovery times) will be applied ANOVA for repeated measurements followed by the Bonferroni post-test for parametric distribution or the Dunnet post-test for non-parametric distribution. The analysis of the different moments between the groups will be made by non-paired Student's t-test for normal distribution data or Mann-Whitney test for non-normal data. The ana- lyses will be performed using the commercial Minitab software - 13.20 version (Minitab, PA., USA) and GraphPad Instat -3.01 version, 1998 (GraphPad Software, Inc., San Diego California USA). P $<0.05$ will be accepted as statistically significant.

\section{Conclusion}

This protocol design would allow to answer important questions and will open new perspectives helping both clinicians and researchers. If the results showed unfavorable impact on autonomic behavior and cardiorespiratory parameters, and that the liquid reposition attenuates this impact, these findings will add an important contribution to cardiovascular rehabilitation program ${ }^{6-11,45}$. Furthermore, to study the recovery period after physical exercise is fundamental since cardiovascular events and sympathovagal unbalance can occur during this per$\operatorname{iod}^{46,47}$.

\section{Acknowledgments}

The authors acknowledgements to Conselho Nacional de Desenvolvimento Científico e Tecnológico - CNPq for financial support in this study. Funding - The study receives financial support of the Conselho Nacional de Desenvolvimento Científico e Tecnológico - CNPq- Grant 401258/2016-5 and Coordenação de Aperfeiçoamento de Pessoal de Nível Superior (CAPES) - Financial code 001.

\section{References}

1. Garber CE, Blissmer B, Deschenes MR, Franklin BA, Lamonte MJ, Lee IM, et al. Quantity and quality of exercise for developing and maintaining cardiorespiratory, musculoskeletal, and neuromotor fitness in apparently healthy adults: Guidance for prescribing exercise. Med Sci Sports Exerc. 2011;43(7):1334-59.

2. Thomas D, Elliott E, Naughton G. Exercise for type 2 diabetes mellitus (Review). Cochrane Database Syst Rev. 2009;(3):1-56.

3. Mccarthy B, Casey D, Devane D, Murphy K, Murphy E, Lacasse Y. Pulmonary rehabilitation for chronic obstructive pulmonary disease (Review). Cochrane Database Syst Rev. 2015;(2):1-298.

4. Lane R, Harwood A, Watson L, Gc L. Exercise for intermittent claudication (Review). Cochrane Database Syst Rev. 2017;(12):1-138.

5. Fuller JT, Hartland MC, Maloney LT, Davison K. Therapeutic effects of aerobic and resistance exercises for cancer survivors?: a systematic review of meta-analyses of clinical trials. Br J Sports Med. 2018;1-11.

6. Anderson L, Thompson D, Oldridge N, Zwisler A, Rees K, Martin N, et al. Exercise based rehabilitation for coronary heart disease ( Review ). Cochrane Database Syst Rev. 2016;(1):1-208.

7. Mandic S, Stevens E, Hodge C, Brown C, Walker R, Body $\mathrm{D}$, et al. Long-term effects of cardiac rehabilitation in 
elderly individuals with stable coronary artery disease. Disabil Rehabil. 2016;38(9):837-43.

8. Laing S, Gluckman T, Weinberg K, Lahiri M, Ng J, Goldberger J. Autonomic effects of exercise based cardiac rehabilitation. J Cardiopulm Rehabil Prev. 2011;31(2):87-91.

9. Rio P, Pereira-da-Silva T, Abreu A, Filipe C, Soares R, Portugal $\mathrm{G}$, et al. Modulating effect of cardiac rehabilitation on autonomic nervous system function in patients with coronary artery disease. Acta Cardiol. 2017;71(6):717-23.

10. Lazzeroni D, Castiglioni P, Bini M, Faini A, Camaiora U, Ugolotti PT, et al. Improvement in aerobic capacity during cardiac rehabilitation in coronary artery disease patients?: Is there a role for autonomic adaptations?? Eur J Prev Cardiol. 2017;24(4):357-64.

11. Besnier F, Labrunée M, Pathak A, Pavy-Le Traon A, Galès $\mathrm{C}$, Sénard JM, et al. Exercise training induced modification in autonomic nervous system: An update for cardiac patients. Ann Phys Rehabil Med. 2017;60(1):27-35.

12. Foss ML, Keteyian SJ. Bases Fisiológicas do Exercício e do Esporte. 2000. 464-77 p.

13. Gonzalez-Alonso J, Mora-Rodríguez R, Below PR, Coyle EF. Dehydration reduces cardiac output and increases systemic and cutaneous vascular resistance during exercise. J Appl Physiol. 1995;79(5):1487-96.

14. Moreno IL, Pastre CM, Papoti M, Vanderlei LCM. Efeitos da reposição hidroeletrolítica sobre parâmetros cardiorrespiratórios em exercício e recuperação. Motriz. 2012;18 (1):165-75.

15. Moreno IL, Pastre CM, Ferreira C, de Abreu LC, Valenti VE, Vanderlei LCM. Effects of an isotonic beverage on autonomic regulation during and after exercise. J Int Soc Sports Nutr. 2013;10(2):1-10.

16. Castro-Sepúlveda M, Cerda-kohler H, Pérez-luco C, Monsalves M, Cristobal D, Zbinden-foncea $\mathrm{H}$, et al. Hydration status after exercise affect resting metabolic rate and heart rate variability. Nutr Hosp. 2015;31(3):1273-7.

17. Carter R, Cheuvront SN, Wray DW, Kolka MA, Stephenson LA, Sawka MN. The influence of hydration status on heart rate variability after exercise heat stress. J Therm Biol. 2005;30(7):495-502.

18. Vanderlei FM, Moreno IL, Vanderlei LCM, Pastre CM, De Abreu LC, Ferreira C. Effects of different protocols of hydration on cardiorespiratory parameters during exercise and recovery. Int Arch Med. 2013;6(1):1-10.

19. Ottone V de O, Magalhães F de C, Paula F de, Avelar NCP, Aguiar PF, Matta PF da, et al. The effect of different water immersion temperatures on post-exercise parasympathetic reactivation. PLoS One. 2014;1-20.

20. Vanderlei FM, Moreno IL, Vanderlei LC, Pastre CM, de Abreu LC, Ferreira C. Comparison of the effect of hydration with water or isotonic solution on the recovery of cardiac autonomic modulation. Int $\mathrm{J}$ Sport Nutr Exerc Metab. 2015;25:145-53.

21. Moreno IL, Vanderlei LCM, Pastre CM, Vanderlei FM, De Abreu LC, Ferreira C. Cardiorespiratory effects of water ingestion during and after exercise. Int Arch Med. 2013;6 (1):1-9
22. Teixeira AL, Ramos PS, Marins JB, Ricardo DR. The role of water intake on cardiac vagal reactivation after upper-body resistance exercise. Int J Sports Med. 2015;36(3):204-8.

23. Vianna LC, Oliveira RB, Silva BM, Ricardo DR, Araújo CGS. Water intake accelerates post-exercise cardiac vagal reactivation in humans. Eur J Appl Physiol. 2008;102 (3):283-8.

24. McDermott BP, Casa DJ, Lee EC, Yamamoto LM, Beasley $\mathrm{KN}$, Emmanuel $\mathrm{H}$, et al. Influence of rehydration mode after exercise dehydration on cardiovascular function. J Strength Cond Res. 2013;27(8):2086-95.

25. La Rovere MT, Bigger JT, Marcus FI, Mortara A, Schwartz PJ. Baroreflex sensitivity and heart-rate variability in prediction of total cardiac mortality after myocardial infarction. Lancet. 1998;351:478-84.

26. Bigger JT, Fleiss JL, Rolnitzky LM, Steinman RC. Frequency domain measures of heart period variability to assess risk late after myocardial infarction. J Am Coll Cardiol. 1993;21(3):729-36.

27. Hamilton MT, Gonzalez-Alonso J, Montain SJ, Coyle EF. Fluid replacement and glucose infusion during exercise prevent cardiovascular drift. J Appl Physiol. 1991;71(3):871-7.

28. Chan A-W, Tetzlaff J, Altman D, Laupacis A, Gøtzsche P, Krleza-Jeric K. Research and reporting methods annals of internal medicine SPIRIT 2013 statement?: defining standard protocol items for clinical trials. Ann Intern Med. 2013;158(3):200-7.

29. Hoffmann TC, Glasziou PP, Boutron I, Milne R, Perera R, Moher D, et al. Better reporting of interventions: Template for intervention description and replication (TIDieR) checklist and guide. BMJ. 2014;348(March):1-12.

30. Godoy MF, Takakura IT, Correa PR. The relevance of nonlinear dynamic analysis (Chaos Theory) to predict morbidity and mortality in patients undergoing surgical myocardial revascularization. Arq em Ciência da Saúde. 2005;12 (4):167-71.

31. Meneghelo RS. III Guidelines of Sociedade Brasileira de Cardiologia on the exercise test. Arq Bras Cardiol. 2010;95 (5 Suppl 1):1-26.

32. Task force of the European society of cardiology of the north American society of pacing electrophysiology. Heart rate variability standards of measurement, physiological interpretation and clinical use. Eur Heart J. 1996;17:354-81.

33. Armstrong L. Hydration assessment techniques. Nutr Rev. 2005;63(6 Pt 2):S40-54.

34. Von Duvillard SP, Braun WA, Markofski M, Beneke R, Leithäuser R. Fluids and hydration in prolonged endurance performance. Nutrition. 2004;20(7-8):651-6.

35. Sawka MN, Burke LM, Eichner ER, Maughan RJ, Montain SJ, Stachenfeld NS. Exercise and fluid replacement. Med Sci Sports Exerc. 2007;39(2):377-90.

36. Barbosa MP da C de R, Silva NT da, Azevedo FM de, Pastre CM, Vanderlei LCM. Comparison of Polar RS800G3 TM heart rate monitor with Polar fi S810i TM and electrocardiogram to obtain the series of RR intervals and analysis of heart rate variability at rest. Clin Physiol Funct Imaging. 2016;36:112-7.

37. Vanderlei LCM, Pastre CM, Hoshi RA, Carvalho TD de, Godoy MF de. Basic notions of heart rate variability and its 
clinical applicability. Rev Bras Cir Cardiov. 2009;24 (2):205-17.

38. Peng CK, Havlin S, Stanley HE, Goldberger AL. Quantification of scaling exponents and crossover phenomena in nonstationary heartbeat time series. Chaos. 1995;5(1):82-7.

39. Marwan N, Romano MC, Thiel M, Kurths J. Recurrence plots for the analysis of complex systems. Phys Rep. 2007;438:237-329.

40. Tarvainen MP, Niskanen JP, Lipponen JA, Ranta-aho PO, Karjalainen PA. Kubios HRV - Heart rate variability analysis software. Comput Methods Programs Biomed. 2014;113 (1):210-20.

41. Goldberger AL, Amaral LAN, Glass L, Hausdorff JM, Ivanov PC, Mark RG, et al. PhysioBank, PhysioToolkit, and PhysioNet components of a new research resource for complex physiologic signals. Circulation. 2000;101:e215-20.

42. Nobre F. VI Brazilian Guidelines for Hypertension. Arquivos Brasileiros de Cardiologia. 2010;95(1):1-51.

43. Irwin S, Tecklin J. Fisioterapia cardiopulmonar. 2003. 348$349 \mathrm{p}$.

44. Borg GA V. Psychophysical bases of perceived exertion. Med Sci Sports Exerc. 1982;15(5):377-81.

45. Godoy M. I Consenso Nacional de Reabilitação Cardiovascular. Arq Bras Cardiol. 1997;69(4):267-91.
46. Chakur BP, Forjaz CLM, Tinucci T, Negrão CE. Adaptações agudas e crônicas do exercício físico no sistema cardiovascular. Rev Paul Educ Física. 2004;18(Esp):21-31.

47. Guyton AC, Hall JE. Textbook of Medical Phyology. 2006. $1128 \mathrm{p}$.

\section{Corresponding author}

Anne Kastelianne França da Silva: Universidade Estadual Paulista, Faculdade de Ciências e Tecnologias, Programa de Pós-Graduação em Fisioterapia Rua Roberto Simonsen 305. Caixa Postal 19060-900, Presidente Prudente, SP, Brazil.

E-mail: anne_franca@hotmail.com.

Manuscript received on February 13, 2020

Manuscript accepted on March 11, 2020

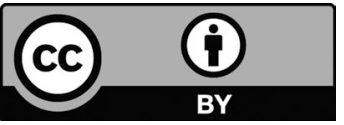

Motriz. The Journal of Physical Education. UNESP. Rio Claro, SP, Brazil - eISSN: 1980-6574 - under a license Creative Commons - Version 4.0 